\title{
Paracetamol: pharmacology, prescribing and controversies
}

\section{Camilla Moriarty, ${ }^{1}$ Will Carroll ${ }^{2}$}

\begin{abstract}
${ }^{1}$ Chelsea and Westminster Hospital, London, UK ${ }^{2}$ Academic Department of Paediatric, Respiratory Medicine, University Hospitals of the North Midlands, Stoke-on-Trent, UK
\end{abstract}

\section{Correspondence to} Dr Will Carroll, Academic Department of Paediatric, Respiratory Medicine, University Hospitals of the North Midlands, Newcastle Road, Stoke-on-Trent ST4 6QG, UK; will.carroll@nhs.net

Received 10 October 2015 Revised 25 March 2016 Accepted 20 April 2016 Published Online First 20 May 2016

\section{CrossMark}

To cite: Moriarty C

Carroll W. Arch Dis Child Educ

Pract Ed 2016;101:331-334.

\section{INDICATIONS AND MECHANISM OF ACTION}

Paracetamol (internationally known as acetaminophen) is the most common medicine encountered in paediatric practice. It is used widely by parents and health professionals and it has analgesic and antipyretic effects. Its short-term safety and efficacy are well established and it is readily available for purchase over the counter. Its mechanism of action is not fully understood but it is known to inhibit prostaglandin synthesis and is highly selective for cyclooxygenase enzymes in the central nervous system. It also has a weak anti-inflammatory action. An understanding of its pharmacology can significantly increase the apparent effectiveness and safety of this useful medicine.

Oral paracetamol is used in children for mild-to-moderate pain and fever, including postimmunisation fever. ${ }^{1}$ It may also be given by intravenous infusion or per rectum when it is most commonly used for the short-term treatment of moderate pain, particularly after surgery, and for persistent fever. Unlike opioid analgesia, there is no dependence or tolerance seen with paracetamol, but there is a ceiling effect on analgesia and once this is achieved higher doses do not have an increased effectiveness. Its long-term use tends to deplete glutathione stores, which can enhance toxicity in overdose. Overdose may result in irreversible hepatotoxicity, which can be fatal.

\section{PHARMACOLOGY OF PARACETAMOL: AN ABCD APPROACH}

Absorption

The pharmacokinetics of paracetamol in infants and children has been described in detail. ${ }^{2}$ Oral formulations have high bioavailability and around $80 \%$ of a dose is absorbed, mainly by passive diffusion. Rectal formulations have a reduced bioavailability compared with other routes and therefore require higher doses.
Importantly, absorption through the gastric mucosa is negligible and therefore any process (or disease) that delays gastric emptying will slow down the absorption of an orally administered dose. The time to peak blood levels after oral administration of the drug in children (aged 6 months to 12 years) ranges from 0.5 to $1.8 \mathrm{~h}$, broadly similar to adults. The presence of food delays the time to peak concentration in the bloodstream, but the overall extent of absorption is unchanged whether food is present or not. It remains unclear as to whether the generally slower gastric emptying time in neonates affects absorption.

\section{Biology}

Despite its ubiquitous usage and a wealth of data on the clinical effects of paracetamol in children and adults, the precise biological mechanism of action remains elusive. It is a weak inhibitor of the synthesis of prostaglandins having clinical effects that are similar but not identical to selective cyclooxygenase-2 (COX-2) inhibitors. However, unlike the selective COX-2 inhibitors, paracetamol does not suppress the inflammation of rheumatoid arthritis.

Paracetamol is widely distributed in the body throughout the majority of body fluids and has a relatively large volume of distribution, which is largest in the preterm infant and reduces with maturity until it reaches adult levels at about 1 year. ${ }^{3}$ It rapidly crosses the blood-brain barrier to the central nervous system; one report found peak cerebrospinal fluid levels in children after $57 \mathrm{~min}$ when administered intravenously. Analgesic activity is produced by the fraction that penetrates into the brain, possibly by an action upon serotonergic pathways. ${ }^{4}$

The clinical effects of oral paracetamol suspension in children often appear to be very rapid in clinical practice. Fever responds quickly and it typically takes 
just over $1 \mathrm{~h}$ to resolve fever in febrile children between 6 months and 6 years of age (a mean of $71 \mathrm{~min}) .^{5}$ In one study, axillary temperature was recorded every $30 \mathrm{~s}$. While the study did not include a placebo arm, axillary temperature began to decline in the paracetamol group within a few minutes of administration. However, the decline in temperature was more rapid in children treated with ibuprofen or ibuprofen and paracetamol in combination. ${ }^{5}$

\section{Clearance}

Paracetamol is primarily metabolised in the liver to glucuronide and sulfate conjugates that are then excreted renally. The sulfation pathway predominates in neonates; the glucuronidation pathway takes time to develop and is mature at around 2 years of age. One study analysed the urinary excretion of the two conjugates $(0-36 \mathrm{~h})$ and found that the ratio of paracetamol-glucuronide to paracetamol-sulfate for neonates (1-2 days old), children (3-9 years) and older children (12 years old) was $0.34,0.75$ and 1.61, respectively, with an adult ratio of 1.80 (see figure 1). ${ }^{6}$

There appears to be no clear clinical consequence of this different pattern of metabolism and by adulthood the predominant pathway is one of glucuronidation. A small proportion of the dose $(5-10 \%)$ is oxidised by CYP450-dependent pathways in the liver by the enzyme CYP2E1. This appears to be present at lower levels in neonates than in adults, leading to the formation of a smaller amount of the toxic intermediate metabolite $\mathrm{N}$-acetyl- $p$-benzoquinone imine (NAPQI). NAPQI is normally detoxified by conjugation with glutathione and the bound NAPQI product is eliminated in the urine or bile. However, if there is not sufficient glutathione available, either as a result of malnutrition or if there is a large amount of NAPQI produced as in overdose, then hepatotoxicity may result (see figure 2).

The elimination half-life for paracetamol varies between neonates, infants, children and adolescents. In neonates born at term it is between 2.5 and $4 \mathrm{~h}$, this is about $1 \mathrm{~h}$ longer than older children. In

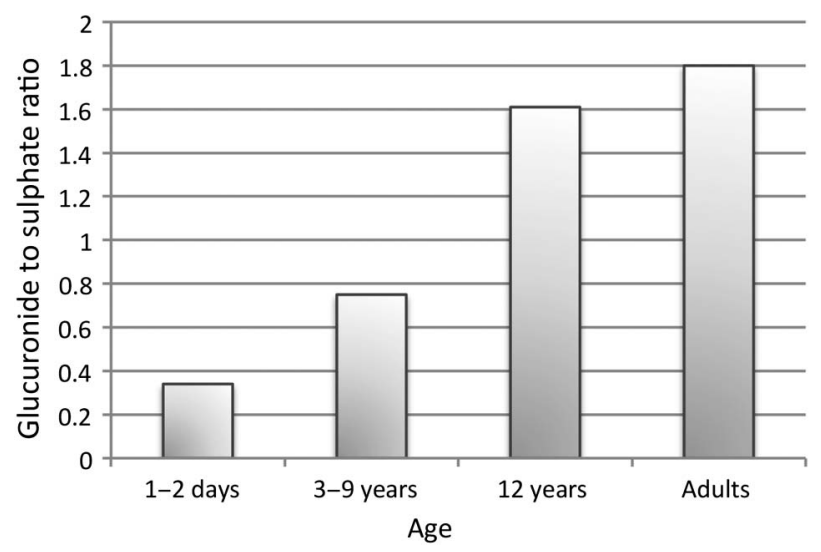

Figure 1 Ratio of glucuronidation to sulfation in different ages. ${ }^{6}$

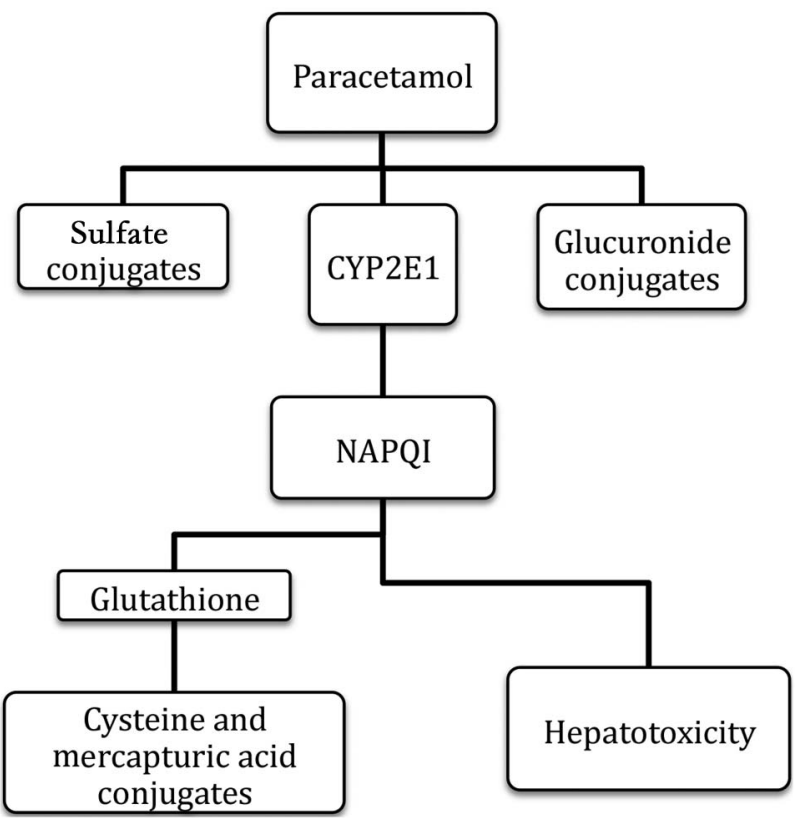

Figure 2 Paracetamol metabolism.

premature neonates, the mean elimination half-life is longer still; one study found it was $11 \mathrm{~h}$ for 28 32 week old neonates (for rectally administered paracetamol) and 4-5 h in 32-36 week old neonates. ${ }^{7}$ The recommended dose frequency in preterm neonates reflects this.

\section{Dosing, indications, practical prescribing issues and controversies in children}

The use of antipyretic medications to achieve a reduction of temperature in children with febrile illness is a controversial area. High temperatures in children cause parental anxiety and this is a common reason for seeking medical attention. Undue emphasis on a need for reduction of fever in children has led to possible overuse of both paracetamol and ibuprofen, often in combination. There is no evidence that this reduces morbidity or mortality. The focus of recent National Institute for Health and Care Excellence (NICE) guidelines ${ }^{8}$ has therefore shifted away from reducing fever to ensuring that the child is comfortable.

There have been concerns that paracetamol will dampen the immune response when used in standard therapeutic doses. Two open-label randomised controlled studies examined the effectiveness and safety of paracetamol suspension following childhood immunisation. These found a blunting of the functional antibody response in the paracetamol group. However, the same authors have repeated this study in children receiving the meningococcal $\mathrm{B}$ vaccine in conjunction with other childhood immunisations and concluded that there is a reduction in postimmunisation fever that is not accompanied by any significant attenuation of the immune response. The 
significance of this possible effect remains unclear. ${ }^{9}$ For the time being, reduction of postvaccination fever remains a licensed indication.

While paracetamol is considered a benign medicine without longer term effects, it has effects on cytokine production that are only partly understood. Some authors have suggested that paracetamol use in pregnancy or early infancy may increase the risk of asthma in young children. A systematic review and meta-analysis of studies reporting this association found that there was insufficient evidence to warrant changing guidelines. ${ }^{10}$

\section{PITFALLS IN PRESCRIBING PARACETAMOL}

Prescribers should beware of the risk of overdosage and should ensure that the appropriate strength of product is prescribed (there are several available). A retrospective study conducted in Australia and New Zealand described paracetamol overdose, due to medication errors, being one of the leading causes of acute liver failure in children. ${ }^{11}$ The common errors encountered in clinical practice are summarised in box 1 . However, this list is far from complete and a recent article has highlighted concerns regarding the lack of research available about the reasons behind medication errors made by caregivers of young children and of the increasing prevalence of falsified medicines used. ${ }^{12}$

Health professionals should ensure that caregivers understand what dose to give and the importance of ensuring that the right strength of product is used. While measuring a dose seems straightforward to a health professional, an untrained carer may have more difficulty; one study of over 270 caregivers aimed to determine the most accurate measuring device by asking carers to measure $5 \mathrm{~mL}$ of $120 \mathrm{mg} / 5 \mathrm{~mL}$ paracetamol using either a $5 \mathrm{~mL}$ metal teaspoon, a $5 \mathrm{~mL}$ calibrated spoon or a $5 \mathrm{~mL}$ oral syringe. Results showed the volumes measured ranged from 0.83 to

Box 1 Errors leading to unintentional paracetamol overdose by caregivers in children ${ }^{11}$

Common errors that led to overdose were:

- Children receiving double doses

- Co-administration of other paracetamol containing preparations

- Exceeding recommended daily dosages

- Increased dose frequency

Reported reasons given by caregivers for these errors were:

- Failure to understand prescriptions

- The use of incorrect measuring devices

- The use of different concentrations of paracetamol

- Lack of recognition of paracetamol in other common 'cold remedies'.
$6.52 \mathrm{~mL}$. The calibrated spoon was the most accurate while the oral syringe had the smallest variance. ${ }^{13}$

In addition, paracetamol is a component of several multi-ingredient medicines sold over the counter. It is important for all involved to understand that the simultaneous administration of one of these, together with prescribed paracetamol may result in inadvertent overdose. When prescribing paracetamol, it is important to try to avoid vague prescribing instructions such as 'take when required'; as errors are more commonly made when instructions are unclear. A minimum time frame between doses and maximum dosing per $24 \mathrm{~h}$ are essential to minimise the risk of overdose.

There remains debate about whether prescribers should use weight or age to determine the appropriate dose of paracetamol and regular prescribers will be aware that the BNF-C has varied this advice over recent years. One study which supported the use of a weight-based dose found that children who were underweight or overweight for their age were at risk of receiving inappropriate doses of paracetamol with the potential of causing harm. ${ }^{14}$ The UK Medicines and Healthcare Regulatory body recognised this

Test your knowledge

1. Which of the following conditions is most likely to result in oral paracetamol being ineffective?

Select ONE answer only:
A. Coeliac disease
B. Hepatic encephalopathy
C. Kawasaki disease
D. Pyloric stenosis
E. Renal failure

2. You are called to the neonatal unit to review a preterm infant of 29 weeks gestation. He is in pain and you plan to prescribe paracetamol.

Which of the following is most likely to be true?

Select ONE answer only:

A. Intravenous administration is safer

B. Most will be metabolised by glucuronidation

C. Paracetamol is unsafe in this child and should be avoided

D. Smaller amounts of the toxic intermediate NAPQI will be formed than in a 1-year-old

E. The elimination half-life is slightly shorter than that of a term infant

3. What is the maximum safe daily dose of oral paracetamol in children?
A. $60 \mathrm{mg} / \mathrm{kg}$
B. $65 \mathrm{mg} / \mathrm{kg}$
C. $70 \mathrm{mg} / \mathrm{kg}$
D. $75 \mathrm{mg} / \mathrm{kg}$
E. $80 \mathrm{mg} / \mathrm{kg}$

Answers are at the end of the references. 
concern and developed new guidance with narrower age ranges for certain doses. ${ }^{15}$ Following concerns about hepatotoxicity at therapeutic doses, there has also been a move to reduce the maximum total daily dose to $75 \mathrm{mg} / \mathrm{kg} /$ day from $90 \mathrm{mg} / \mathrm{kg} /$ day. Prescribers should always seek help and advice if they are at all unsure of what dose to use and even experienced prescribers are advised to check the latest version of the BNF-C from time to time to keep up to date with changes.

\section{SUMMARY}

Paracetamol is a useful medicine. Its benefits as an analgesic and antipyretic are widely known but it should be used with caution and an appreciation of its mechanism of action. It works optimally when the right dose is given by the right route. As with all medicines, adverse effects can (and do) occur as a result of poor prescribing and following accidental or purposeful ingestion. There may be longer term effects on general health that should not be overlooked-and it certainly should not be simply 'prescribed routinely' to all children admitted to hospital or given indiscriminately to all children who are febrile.

Contributors CM and WC jointly prepared the review and contributed equally to the final version.

Competing interests None declared.

Provenance and peer review Commissioned; externally peer reviewed.

\section{REFERENCES}

1 Paediatric Formulary Committee. BNF for children. London: BMJ Group, Pharmaceutical Press, and RCPCH Publications. http://www.medicinescomplete.com (accessed 2 May 2015).

2 JI P, Wang Y, Li Z, et al. Regulatory review of acetaminophen clinical pharmacology in young pediatric patients. J Pharm Sci 2012;101:4383-9.

3 Anderson BJ, van Lingen RA, Hansen TJ, et al. Acetaminophen developmental pharmacokinetics in premature neonates and infants: a pooled population analysis. Anesthesiology 2002;96:1336-45.

4 Kumpulainen E, Kokki H, Halonen T, et al. Paracetamol (Acetaminophen) penetrates readily into the cerebrospinal fluid of children after intravenous administration. Pediatrics 2007;119:766-71.

5 Hay AD, Costelloe C, Redmond NM, et al. Paracetamol plus ibuprofen for the treatment of fever in children (PITCH): randomised controlled trial. BMJ 2008;337:a1302.

6 van der Marel CD, Anderson BJ, van Lingen RA, et al. Paracetamol and metabolite pharmacokinetics in infants. Eur J Clin Pharmacol 2003;59:243-51.

7 van lingen RA, Deinum JT, Quak JM, et al. Pharmacokinetics and metabolism of rectally administered paracetamol in preterm neonates. Arch Dis Child Fetal Neonatal Ed 1999;80:FF9-63.

8 National Collaborating Centre for Women's and Children's Health. Feverish illness in children: assessment and initial management in children younger than 5 years (full NICE guideline). Clinical guidance 160 National Centre for Health and Care Excellence. 2013. https://www.nice.org.uk/guidance/ cg160 (accessed 7 Aug 2015).

9 Prymula R, Siegrist CA, Chlibek R, et al. Effect of prophylactic paracetamol administration at time of vaccination on febrile reactions and antibody responses in children: two open-label, randomised controlled trials. Lancet 2009;374: 1339-50.

10 Cheelo M, Lodge CJ, Dharmage SC, et al. Paracetamol exposure in pregnancy and early childhood and development of childhood asthma: a systematic review and meta-analysis. Arch Dis Child 2015;100:81-9.

11 Rajanayagam J, Bishop JR, Lewindon PJ, et al. Paracetamol-associated acute liver failure in Australian and New Zealand children: high rate of medication errors. Arch Dis Child 2015;100:77-80.

12 Star K, Choonara I. How safe is paracetamol? Arch Dis Child 2014;100:73-4.

13 Beckett VL, Tyson LD, Carroll D, et al. Accurately administering oral medication to children isn't child's play. Arch Dis Child 2012;97:838-41.

14 Eyers S, Fingleton J, Eastwood A, et al. British National Formulary for Children: the risk of inappropriate paracetamol prescribing. Arch Dis Child 2012;97: 279-82.

15 Lenney W. Editorial: Paracetamol prescription by age or by weight? Arch Dis Child 2012;97:277-8.

\section{Answers to the quiz questions}

1. Answer-D. Pyloric stenosis

Rationale: Paracetamol is not effectively absorbed from the stomach. Therefore, in any condition that results in no (or substantially delayed) gastric emptying it will be ineffective as both an antipyretic or analgesic.

2. Answer-D.

Smaller amounts of the toxic intermediate NAPQI will be formed than in a 1-year-oldRationale: A small proportion of the dose (5-10\%) is oxidised by CYP450-dependent pathways in the liver by the enzyme CYP2E1. This appears to be present at lower levels in neonates than in adults, leading to the formation of a smaller amount of the toxic intermediate metabolite NAPQI. Although paracetamol has a longer half-life in neonates, the elimination is mostly through sulfation and it is relatively safe. In order to be effective, the stomach must be emptying but there is no evidence that intravenous paracetamol is safer.

3. Answer-D. $75 \mathrm{mg} / \mathrm{kg}$

Rationale: Following concerns about hepatotoxicity at therapeutic doses, there has also been a move to reduce the maximum daily dose to $75 \mathrm{mg} / \mathrm{kg} /$ day from $90 \mathrm{mg} / \mathrm{kg} /$ day. 